\title{
Patient specific ankle-foot orthoses using rapid prototyping
}

\author{
Constantinos Mavroidis ${ }^{1 *}$, Richard G Ranky ${ }^{1}$, Mark L Sivak ${ }^{1}$, Benjamin L Patritti ${ }^{2}$, Joseph DiPisa', Alyssa Caddle ${ }^{1}$, \\ Kara Gilhooly ${ }^{1}$, Lauren Govoni ${ }^{1}$, Seth Sivak', Michael Lancia ${ }^{3}$, Robert Drillio ${ }^{4}$, Paolo Bonato ${ }^{2,5^{*}}$
}

\begin{abstract}
Background: Prefabricated orthotic devices are currently designed to fit a range of patients and therefore they do not provide individualized comfort and function. Custom-fit orthoses are superior to prefabricated orthotic devices from both of the above-mentioned standpoints. However, creating a custom-fit orthosis is a laborious and timeintensive manual process performed by skilled orthotists. Besides, adjustments made to both prefabricated and custom-fit orthoses are carried out in a qualitative manner. So both comfort and function can potentially suffer considerably. A computerized technique for fabricating patient-specific orthotic devices has the potential to provide excellent comfort and allow for changes in the standard design to meet the specific needs of each patient.
\end{abstract}

Methods: In this paper, 3D laser scanning is combined with rapid prototyping to create patient-specific orthoses. A novel process was engineered to utilize patient-specific surface data of the patient anatomy as a digital input, manipulate the surface data to an optimal form using Computer Aided Design (CAD) software, and then download the digital output from the CAD software to a rapid prototyping machine for fabrication.

Results: Two AFOs were rapidly prototyped to demonstrate the proposed process. Gait analysis data of a subject wearing the AFOs indicated that the rapid prototyped AFOs performed comparably to the prefabricated polypropylene design.

Conclusions: The rapidly prototyped orthoses fabricated in this study provided good fit of the subject's anatomy compared to a prefabricated AFO while delivering comparable function (i.e. mechanical effect on the biomechanics of gait). The rapid fabrication capability is of interest because it has potential for decreasing fabrication time and cost especially when a replacement of the orthosis is required.

\section{Background}

The unique advantages of rapid prototyping (RP) (also called layered manufacturing) for medical application are becoming increasingly apparent. Furthermore, developments in $3 \mathrm{D}$ scanning have made it possible to acquire digital models of freeform surfaces like the surface anatomy of the human body. The combination of these two technologies can provide patient-specific data input corresponding to anatomical features (via

\footnotetext{
* Correspondence: mavro@coe.neu.edu; PBONATO@PARTNERS.ORG 'Department of Mechanical \& Industrial Engineering, Northeastern University, 360 Huntington Avenue, Boston, MA, 02115, USA

${ }^{2}$ Department of Physical Medicine and Rehabilitation, Harvard Medical School, Spaulding Rehabilitation Hospital, 125 Nashua Street, Boston, MA, 02114, USA

Full list of author information is available at the end of the article
}

3D scanning), as well as a means of producing a patientspecific form output (via RP). Both technologies appear to be ideally suited for the development of patientspecific medical appliances and devices such as orthoses.

This paper details a novel process that combines 3D laser scanning with RP to create patient-specific orthoses. The process was engineered to utilize surface data of the patient anatomy as a digital input, manipulate the surface data to an optimal form using Computer Aided Design (CAD) software, and then download the digital output from the CAD software to a RP machine for fabrication. The methods herein presented have the potential to ultimately provide increased freedom with geometric features, cost efficiencies and improved practice service capacity while maintaining high quality-ofservice standards.

\section{() Biomed Central}




\section{D Scanning Technologies for Medical Modeling}

Medical modeling is a process by which a particular part of the human body is re-created in the form of an anatomically correct digital model first and then as a physical prototype/model. Such models have had successful implementation in preoperative planning, implant design/fabrication, facial prosthetics post-surgery and teaching/concept communication to patients or medical students [1-3].

There are several 3D scanning technologies used to input the data necessary for medical modeling. Laser scanning is one method of capturing the anatomical data needed to create these models as exact replicas of the human body. 3D laser scanners use a laser beam normal to the surface to be scanned. The light reflected back from the surface is captured as a $2 \mathrm{D}$ projection by a CCD (charged-couple device) camera and a point cloud is created using a triangulation technique.

A second type of 3D scanner is based upon stereoscopic photogrammetry. 3D photogrammetric scanners use images captured from different points of view. Given the camera locations and orientations, lines are mathematically triangulated to produce 3D coordinates of each unobscured point in both pictures necessary to reproduce an adequate point cloud for shape and size reproduction.

Software packages that are used to create medical models for RP are unique in that they must take information from a $2 \mathrm{D}$ scan of the body and use that information to create a $3 \mathrm{D}$ model. They also have CAD functionalities to provide the possibility of optimizing the design of the model based on the application needs. The output file from the data analysis and design software is written in the standard tessellation language (STL) format, which is the most common file type used with RP machines. Once the human anatomy has been recorded and a digital model has been created, the produced STL file instructs the RP machine about how to manufacture the intended medical model $[4,5]$.

\section{Rapid Prototyping for Medical Modeling and Rehabilitation}

RP has been extensively used in medicine [6]. Depending on the anatomy that is being modeled and the application of interest, different types of RP machines may be most appropriate.

The most broadly used RP technique for surgical planning and training is stereolithography (SLA) [7]. An SLA machine uses a laser beam to sequentially trace the cross sectional slices of an object in a liquid photopolymer resin. The area of photopolymer that is hit by the laser partially cures into a thin sheet. The platform upon which this sheet sits is then lowered by one layer's thickness (resolution on the order of $0.05 \mathrm{~mm}$ ) and the laser traces a new cross section on top of the first layer. These sheets continue to be built one on top of another to create the final three-dimensional shape. Some of the advantages of SLA are its high accuracy, the ability to build clear models for examination, and - with some materials - sterilization for biocompatibility.

Another RP technique known to the medical field is selective laser sintering (SLS) [e.g. 8]. This technology is similar to SLA since it relies upon a laser to sketch out the region to be built on a substrate. In this process, however, the laser binds a powder substrate rather than curing a liquid. This powder is typically rolled over the layer built before it by precision rollers, and each layer is dropped down exposing an area for a second layer to be applied. This technology can utilize stainless-steel, titanium, or nylon powders as fabrication materials.

In rehabilitation, RP has been used for the fabrication of prosthetic sockets $[9,10]$. It has been also proposed as a way to optimize the design of customized rehabilitation tools [11]. Research on the development of customfit orthoses using RP has been very limited. A 3D scanner in conjunction with SLS was used by Milusheva et al. [12,13] to develop 3D models of customized AFO's. However, the SLS prototype of the customized AFO was used only for design evaluation purposes and not as the functional prototype. Another customized AFO manufactured using SLS was presented by Faustini et al. [14]. The geometry of these AFOs was captured by Computed-Tomography (CT) scanning of an AFO built using a conventional technique rather than generating the surface model directly from the subject's anatomy.

It is clear that although some important pioneering research has already been performed in the area of RP patient-specific orthoses, several aspects of the implementation of the technique to manufacture AFOs using RP need to be addressed including: a) demonstrating the full design/manufacturing cycle starting from obtaining scans of the human anatomy to fabricating the customized orthosis; and b) performing gait analysis experiments to evaluate the mechanical effect of orthoses manufactured using RP and compare their performance with that achieved using orthoses fabricated by means of conventional techniques.

\section{Current Methodology to Develop Custom-Fit AFOs}

Creating a custom-fit AFO is a laborious and timeintensive manual process performed by skilled orthotists. This process is depicted in Figure 1 and can take up to 4 hours of fabrication time per unit for an experienced technician. Once the orthotist has determined the configuration and orientation of the subject's anatomy for corrective measures, the form is captured by wrapping a sock and casting the leg (Figure 1a). Markings are drawn at key locations onto the sock surface which 


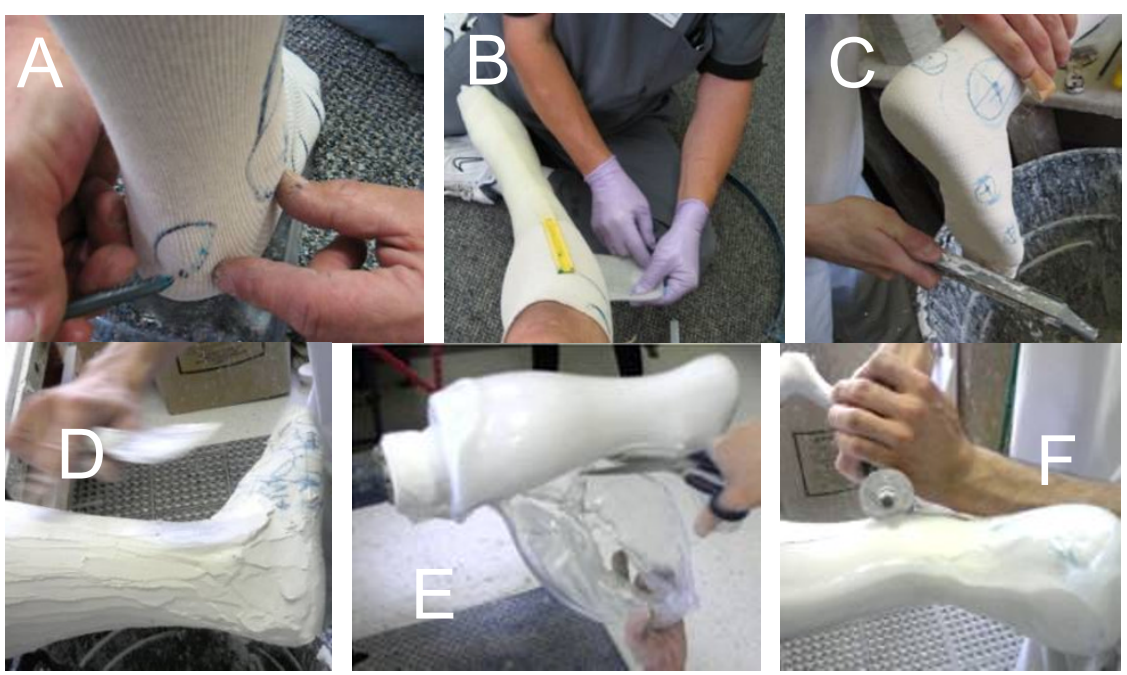

Figure 1 Traditional fabrication process of an ankle foot orthosis for a patient.

instruct technicians later on about where to perform corrective modifications. Once the cast has set (Figure 1b), it is cut away along the anterior contour, in line with the tibia (Figure 1c). The open edge of the cast is filled and plaster is poured into the leg cavity. Starting at the heel, key surfaces are built outwards with plaster by embedding staples corresponding to surface markers (Figure 1d). Once the leg bust has been modified, preheated thermoplastic is vacuum formed around the plaster (Figure 1e). Once cool, the unwanted plastic is cut away, leaving an uneven 1/4" deep gash in the modified leg bust, and requiring edges on the AFO to be ground down \& smoothed (Figure 1f). The back vertical surface of the removed AFO is loaded and bent forward by the technician to check for even splay during weight bearing. Should the need arise to re-fabricate a patient's AFO, the gash in the bust must be repaired before thermoforming can take place. Due to warehousing considerations, most leg busts in clinics are not kept for more than typically 2 months, so for each patient refitting (typically occurring every other year), the whole process must start from the beginning.

\section{Methods}

The main steps of the proposed method are: a) positioning the patient in a way that is suitable for scanning and taking the scan using a 3D scanner that is capable of creating a full $3 \mathrm{D}$ point cloud of the ankle-foot complex (or any other joint of interest); b) processing and manipulating the data from the scan to create the computer model of the desired orthosis including performing design modifications to optimize the shape of the orthosis according to the clinical needs; c) fabricating the custom-fit orthosis using a RP machine. Figure 2 illustrates the process.

To show that the proposed technique can lead to manufacturing an AFO comparable to a prefabricated one, we chose a posterior leaf spring AFO (Type C-90 Superior Posterior Leaf Spring, AliMed, Inc., Dedham, MA) as an exemplary orthotic device to be matched by using the proposed RP-based technique [15]. The RP implementation of the posterior leaf spring AFO used a 3D FaceCam 500 from Technest Inc. [16] for acquiring the data of the human's anatomy and a Viper Si2 SLA machine from 3D Systems Inc. for layered manufacturing [17].

\section{D Scanning}

The 3D FaceCam 500 scanner from Technest Inc captures three images (two for surface shape, one for color) with a resolution of $640 \times 480$ pixels. During a scan, a pattern of colored light is projected onto the target surface. The reflected light from this pattern is captured by camera lenses at two different locations, which will later be used to reconstruct the shape digitally. In order to get the most accurate data possible from the $3 \mathrm{D}$ scans, a procedure was developed for scanning a subject's ankle and foot. The design required data from below the knee and to the posterior of the leg and also the ventral side of the foot. The camera locations for scans are dictated by its range and field of view, which directly impact the quality of the data. The scanning operation was broken down into 3 vertical images of the ankle region and 3 images of the bottom of the foot whilst the subject was not load bearing. A white background was placed around the leg to differentiate the subject's leg from 


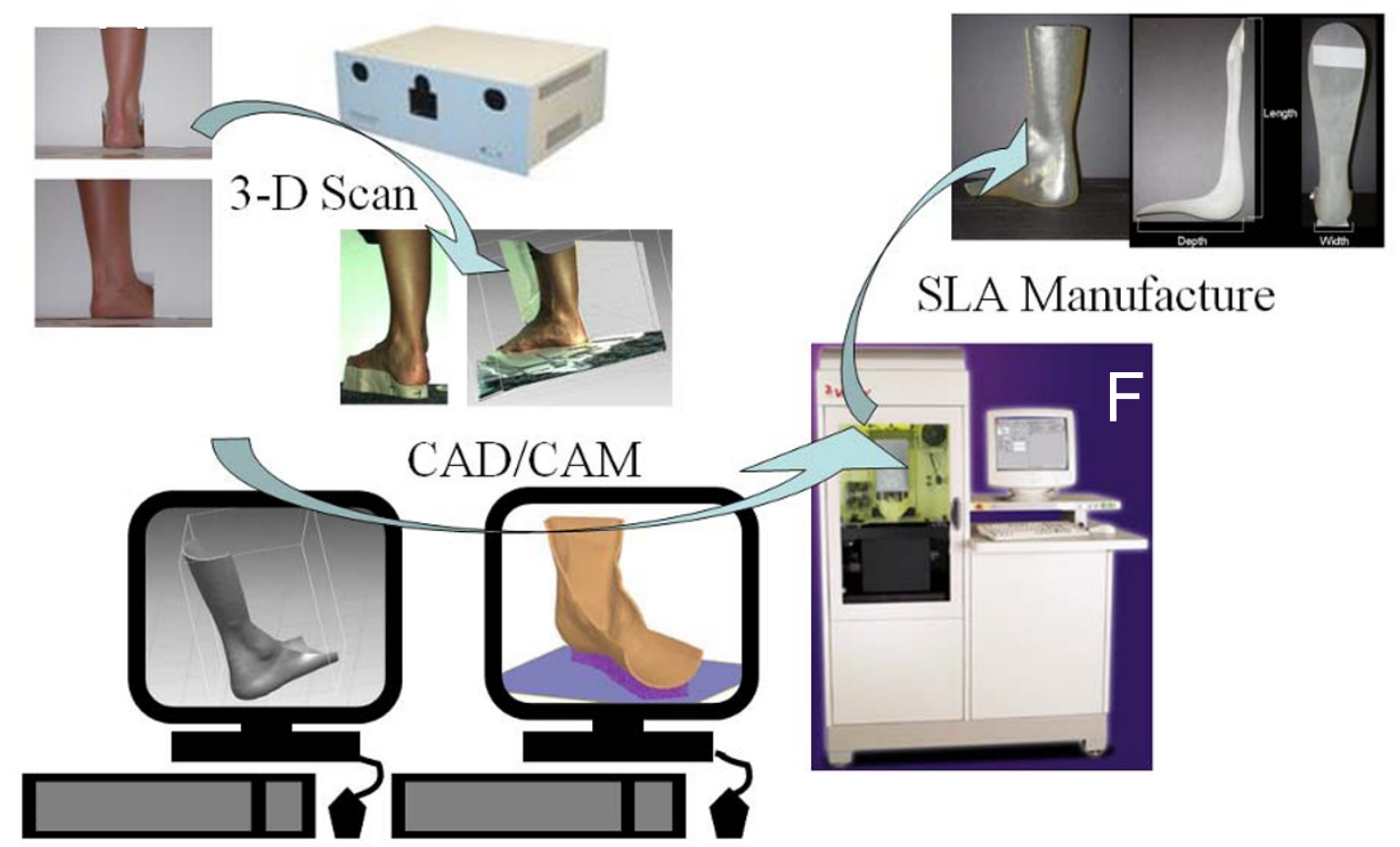

Figure 2 Process used to fabricate the proof of concept AFOs

extraneous data. Figure 3 shows the position of the camera for each of the scans of the ankle-foot complex while load bearing and one view of the subject's foot and ankle as seen by the 3D scanner. The non-load bearing scans were taken with the knee at about 90 deg and the shank in a vertical position.

\section{Software}

The acquired scans were post-processed using the software Rapidform [18]. This software was used to clean and convert the scans by removal of unwanted points and meshing of the point cloud into a single shell. Figure 4 illustrates this process.

The process began with removing redundant data points (Figure 4). This includes data from the parts of the leg that were not needed as well as mismatching surfaces and data from the floor or background for each captured view. The points within each cloud were then connected to each other with three-sided polygons to create a surface mesh. The individual surface meshes
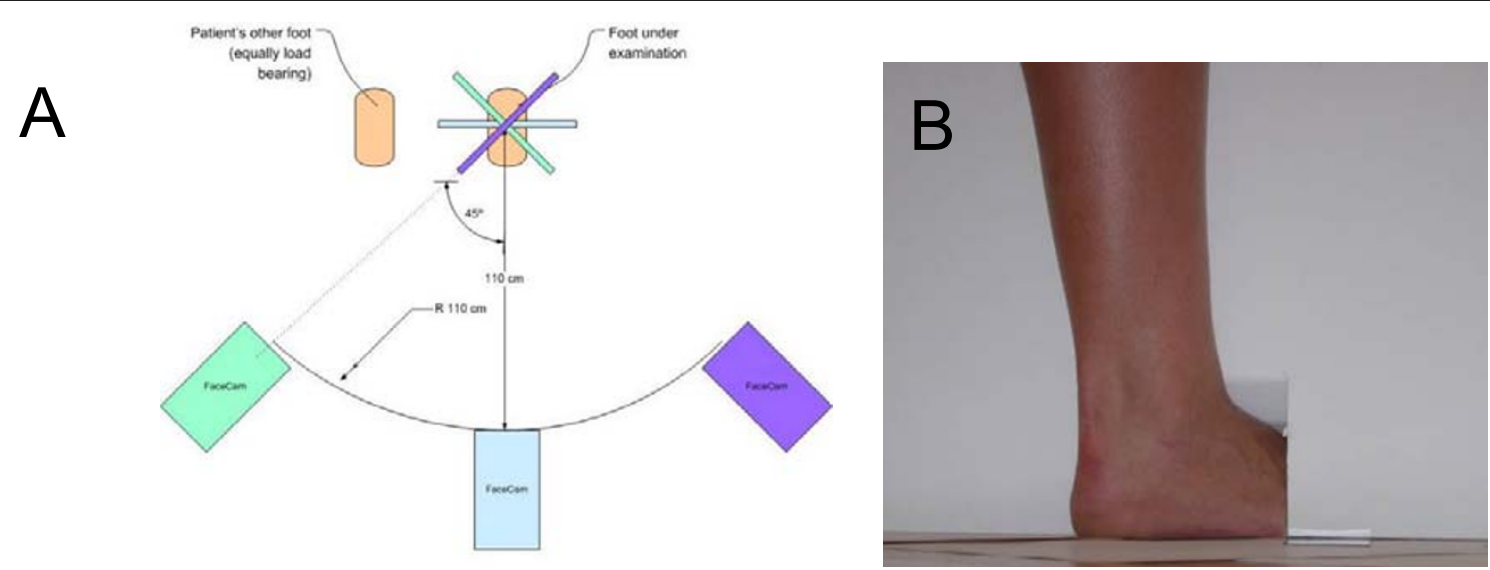

Figure 3 Positioning of the foot during laser scanning. (A) Schematic of the setup and procedure used to scan the ankle of the subject. Note the relative positions of the cameras. (B) Lateral aspect of the foot and ankle as seen from the perspective of the right camera of the scanner. 


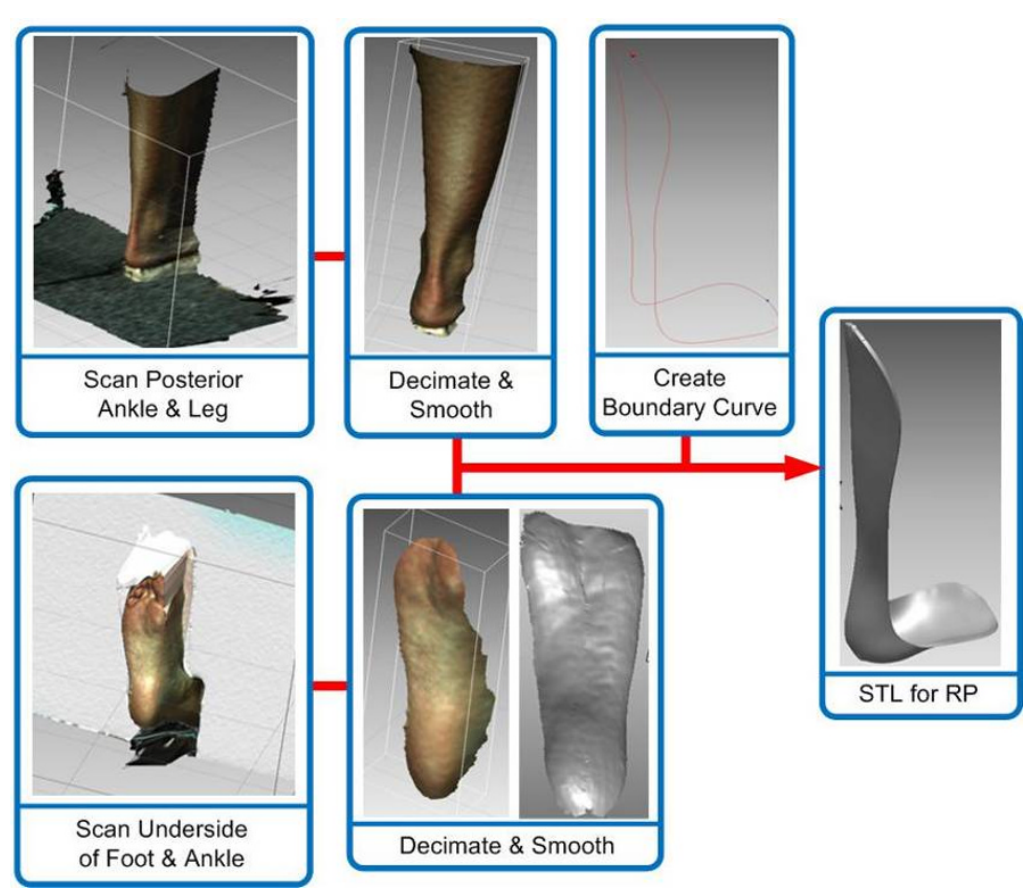

Figure 4 Flow diagram of the post-scanning software procedures.

were aligned and merged to create one complete surface model of the ankle-foot complex. The polygon surface curvature was smoothened and edges then trimmed with a boundary curve. This surface was then offset to prevent the fabricated AFO from over-compressing the subject's leg. The offset surface was extruded to a thickness of $3 \mathrm{~mm}$ as typically done for fitting of standard AFOs [15]. Once completed, the model was exported from Rapidform as a STL file.

\section{Rapid Prototyping}

The model was manufactured using the 3D Systems Viper Si2 SLA machine [17]. This system uses a solid

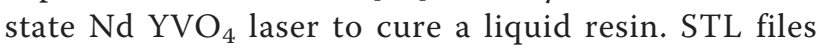
were prepared with $3 D$ Lightyear for part and platform settings, and Buildstation to optimize the machine's configuration.

The effectiveness of using RP for the application at hand is largely dependent on material properties. The prefabricated AFO selected for the study (i.e. the one we attempted to match using the proposed methodology based on RP) was the Type C-90 Superior Posterior Leaf Spring from AliMed [15]. This AFO comes in a pre-determined range of sizes of injection molded polypropylene.

Two different AFOs, each fabricated with a different material, were built using the Viper SLA machine. The first material was the Accura 40 resin that produced a rigid AFO while the second AFO was more flexible as it was manufactured using the DSM Somos 9120 Epoxy Photopolymer. This resin is biocompatible for superficial exposure and offers good fatigue properties relative to the polypropylene [19]. Material properties are compared in Table 1.

\section{Gait Analysis}

Gait studies were conducted at Spaulding Rehabilitation Hospital, Boston, MA using a motion capture system. We collected data from a healthy subject (the one for which scans were taken in order to manufacture the AFO) walking without an AFO, walking with the abovementioned standard, prefabricated AFO, and walking with each of the AFOs manufactured using the

\section{Table 1 AFO material properties}

\begin{tabular}{|c|c|c|c|}
\hline Description & $\begin{array}{c}\text { Unfilled } \\
\text { Polypropylene }\end{array}$ & Accura SI 40 & $\begin{array}{l}\text { Somos }^{\circledR} \\
9120 \text { UV }\end{array}$ \\
\hline $\begin{array}{l}\text { Tensile Strength } \\
\text { (MPa) }\end{array}$ & $31-37.2$ & $57.2-58.7$ & $30-32$ \\
\hline Elongation (\%) & $7-13$ & $4.8-5.1$ & $15-25 \%$ \\
\hline $\begin{array}{l}\text { Young's Modulus } \\
\text { (GPa) }\end{array}$ & $1.1-1.5$ & $2.6-3.3$ & $1.2-1.4$ \\
\hline $\begin{array}{l}\text { Flexural Strength } \\
\qquad(\mathrm{MPa})\end{array}$ & $41-55$ & $93.4-96.1$ & $41-46$ \\
\hline $\begin{array}{l}\text { Flexural Modulus } \\
\qquad(\mathrm{MPa})\end{array}$ & $1172-1724$ & $2836-3044$ & $1310-1455$ \\
\hline
\end{tabular}

a) polypropylene used with the standard, prefabricated AFO); b) Accura SI 40 used with the rigid RP AFO and c) the epoxy photopolymer Somos 9120 used with the flexible RP AFO. 
proposed RP-based technique. The subject wore the AFOs on the right side. Four different conditions were therefore tested: 1) with sneakers and no AFO (No AFO); 2) with the standard, prefabricated polypropylene AFO (Standard AFO); 3) with the rigid AFO made with the Accura 40 resin (Rigid RP AFO), and 4) with the flexible AFO made from the Somos 9120 resin (Flexible RP AFO).

Reflective markers were placed on the following anatomical landmarks: bilateral anterior superior iliac spines, posterior superior iliac spines, lateral femoral condyles, lateral malleoli, second metatarsal heads, and the calcanei (Figure 5). Additional markers were also rigidly attached to wands and placed over the midfemur and mid-shank. The subject was instructed to ambulate along a walkway at a comfortable speed for all of the walking trials. An 8-camera motion capture system (Vicon 512, Vicon Peak, Oxford, UK) recorded the three-dimensional trajectories of the reflective markers during the walking trials. Two force platforms (AMTI OR6-7, AMTI, Watertown, MA) embedded in the walkway recorded the ground reaction forces and moments. Data was gathered at $120 \mathrm{~Hz}$. Ten walking trials with foot contacts of each foot onto the force platforms were collected for each testing condition.

Gait parameters derived from the walking trials included spatio-temporal parameters and kinematics and kinetics of the hip, knee and ankle of each leg in the sagittal plane. Kinematics (joint angles) and kinetics (joint moments and

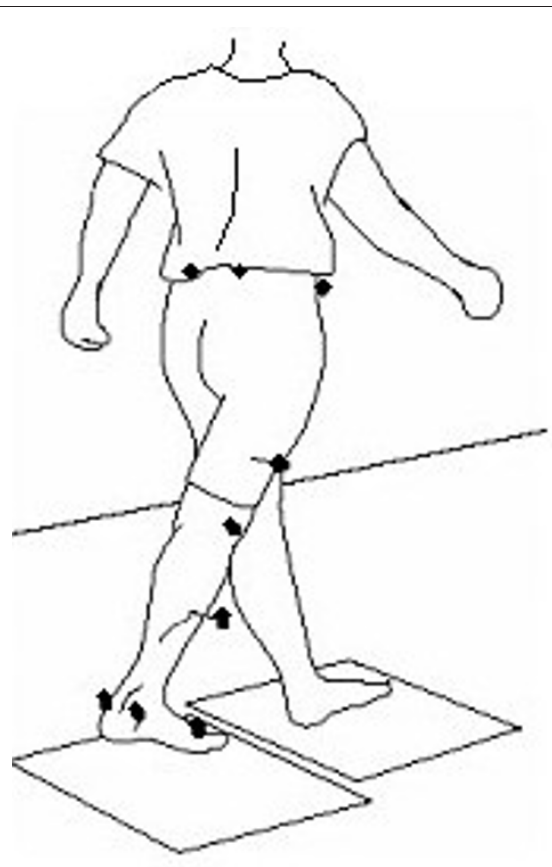

Figure 5 Position of the reflective markers used during the gait analyses. powers) were estimated using a standard model (Vicon Plug-in-Gait, Vicon Peak, Oxford, UK).

\section{Results \\ AFO Fabrication}

The prototype built using the Acura 40 resin is shown in Figure 6. The model had to be built in an inclined orientation since it did not fit sideways (Figure 6A). The build cycle consisted of 2,269 layers of resin and was built in the total time of 16.7 hours due to the large z-build dimension. Fitting of the rigid RP AFO prototype was excellent.

A second prototype was built from the same STL model file but using a more flexible SOMOS 9120 resin. The dimensions of the final prototype AFO and the prefabricated AFO were very closely matched. The weight of the flexible RP AFO was lower by $21 \%$. Figure $7 \mathrm{~A}$ shows the flexible RP AFO. Figure 7B shows the flexible RP AFO being worn by the subject recruited for the scanning. The optimal fit of the AFO geometry to the human subject anatomy was evident from visual inspection and the subject expressed great comfort whilst wearing it.

\section{Testing and Validation}

Analysis of the spatio-temporal gait parameters showed that the subject walked very consistently across the four testing conditions. Differences between the conditions based on the range (minimum and maximum values) of each parameter for the left and right leg were less than $10 \%$. When comparing only the right side, on which the AFOs were worn, the differences between conditions for each of the parameters reduced to $5 \%$ or less (Table 2). This indicates that observed changes in the kinematics and kinetics of gait are likely due to differences in the properties and behavior of the AFOs rather than to fluctuations in speed or step length of the subject during the walking trials for each condition.

The ankle kinematics showed the effect of the three tested AFOs. Figure 8A shows the mean plantarflexiondorsiflexion trajectory of the right ankle for one gait cycle collected during the walking trials performed without AFO. This pattern is typical of individuals without gait abnormalities. For the sake of analyzing the ankle biomechanics, we divided the gait cycle into four subphases (see Figure 8A): controlled plantarflexion (CP) after initial contact, controlled dorsiflexion (CD) as the lower leg progresses forward over the foot, power plantarflexion during push-off (PP), and dorsiflexion during swing (SD) to assist foot clearance. The use of an AFO affected the ankle trajectory during these phases (see Figure 8B). Using the above-defined sub-phases, we compared the movement of the right ankle for the four testing conditions (see Figure $8 \mathrm{~B}$ ) to assess the 


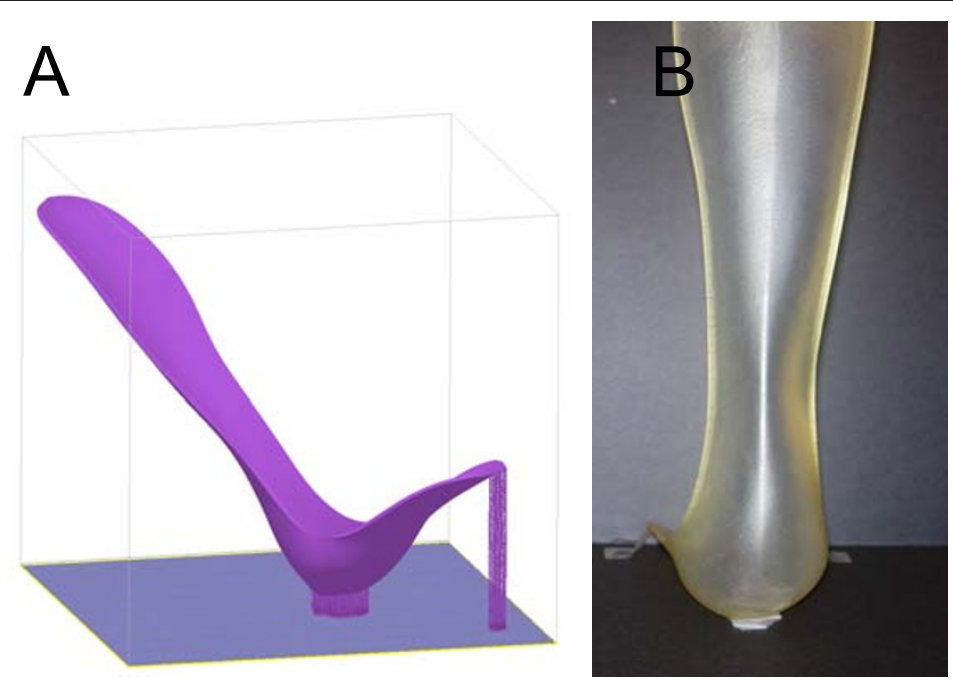

Figure 6 Rigid RP AFO. (A) Example of the build platform. (B) Completed rigid RP AFO prototype.

performance of the three AFOs (prefabricated AFO, Flexible RP AFO, and Rigid RP AFO) and compare the observed kinematic trajectories with the data gathered without using an AFO.

Figure $8 \mathrm{~B}$ shows that the ankle is slightly more plantarflexed at initial contact when wearing no AFO compared to wearing an AFO, and that for each of the AFO conditions initial contact was made with the ankle-foot complex in a more neutral position. This is likely due to the AFOs being made from castings and scans, respectively, of the subject's foot set in a neutral position. During controlled plantarflexion $(\mathrm{CD})$ the ankle showed a similar range of motion (RoM) for each of the AFOs with the standard, prefabricated AFO allowing slightly more plantarflexion compared to the RP AFOs (Figure $8 C)$. This may be due to greater compliance of the polypropylene material from which the standard AFO was made.

During the phase of controlled dorsiflexion (CD), the standard AFO allowed more RoM compared to the two RP AFOs, which performed similarly (Figure 8D). This greater RoM was due to a combination of greater plantarflexion during the $\mathrm{CP}$ phase and also greater dorsiflexion during the $\mathrm{CD}$ phase.

The ankle showed the greatest RoM during the power plantarflexion (PP) phase at push-off when the subject was wearing no brace since the movement of the ankle was not restricted by an AFO. When wearing the AFOs, the amount of plantarflexion was substantially decreased (Figure $8 \mathrm{~B}$ ) while the RoM during the PP phase was slightly greater for the standard AFO compared to the two RP AFOs (Figure 8E).
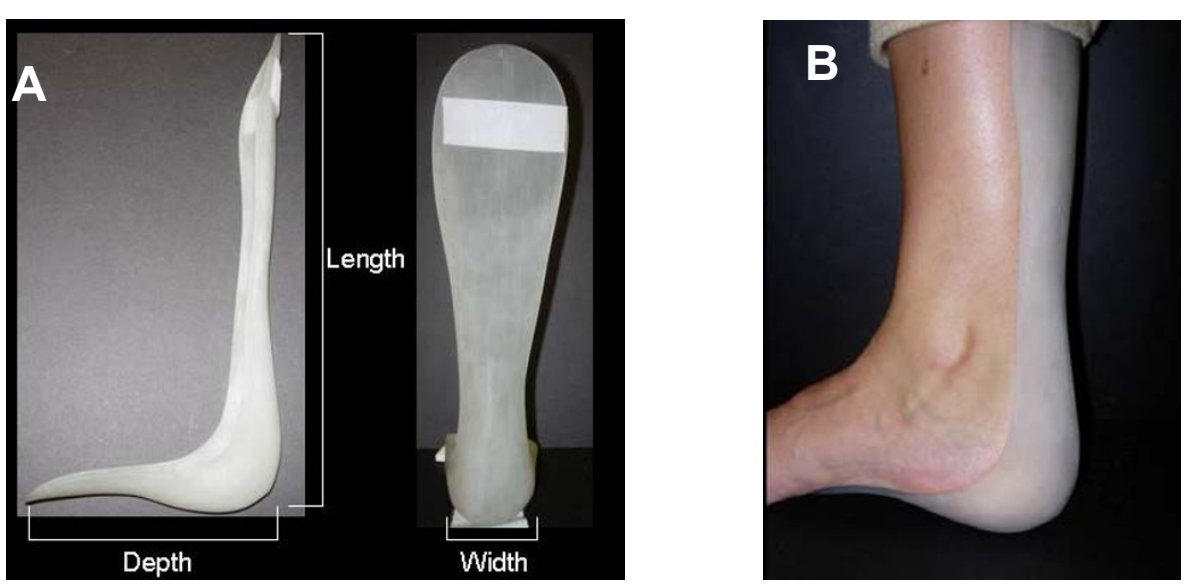

Figure 7 Flexible RP AFO. A) The flexible RP AFO. (B) The positioning and fitting of the flexible RP AFO to the leg of the subject. 
Table 2 Mean ( \pm SD) spatiotemporal gait parameters of the right side for the 4 testing conditions

\begin{tabular}{lcccc}
\hline Parameter & No AFO & Standard AFO & Flexible RP AFO & Rigid RP AFO \\
\hline Walking speed (m/s) & $1.49 \pm 0.05$ & $1.46 \pm 0.02$ & $1.44 \pm 0.05$ & $1.50 \pm 0.06$ \\
Step length (m) & $0.79 \pm 0.02$ & $0.79 \pm 0.01$ & $0.79 \pm 0.03$ & $0.82 \pm 0.03$ \\
Double support time (s) & $0.22 \pm 0.02$ & $0.24 \pm 0.01$ & $0.24 \pm 0.01$ & $0.23 \pm 0.01$ \\
\hline
\end{tabular}

In the final phase of dorsiflexion during swing (SD), the ankle showed the greatest RoM when it was not restricted by an AFO, while the three AFO testing conditions showed lower but similar ranges of motion (Figure 8F). This was partly due to the reduced amount of plantarflexion achieved during the PP phase. Importantly the two RP AFOs enabled a similar amount of ankle dorsiflexion at the end of swing as that allowed by the standard AFO (Figure 8B).

The kinetics of the ankle (joint moments and powers) also revealed that the two RP AFOs performed similarly to the standard AFO. Figure 9A shows the mean right ankle flexion/extension moment during the walking trials for each testing condition. It is evident that the ankle moment profile for the three AFOs was similar.
The peak flexor moment for each AFO testing condition was slightly smaller than that for the no-AFO testing condition (Figure 9B). When comparing the profiles of ankle power, we observed similarities across the three AFO testing conditions (Figure 10) with a general reduction in peak power generation compared to the no-AFO condition. This attenuated peak power is likely due to the restricted plantarflexion of the ankle during push off imposed by the AFOs.

Overall, when comparing the three AFOs, it was clear that they performed similarly in terms of controlling ankle kinematics and kinetics during the gait cycle.

The flexible RP AFO performed almost identically to the standard AFO. Both required less ankle power than normal (i.e. with no AFO). The rigid AFO results

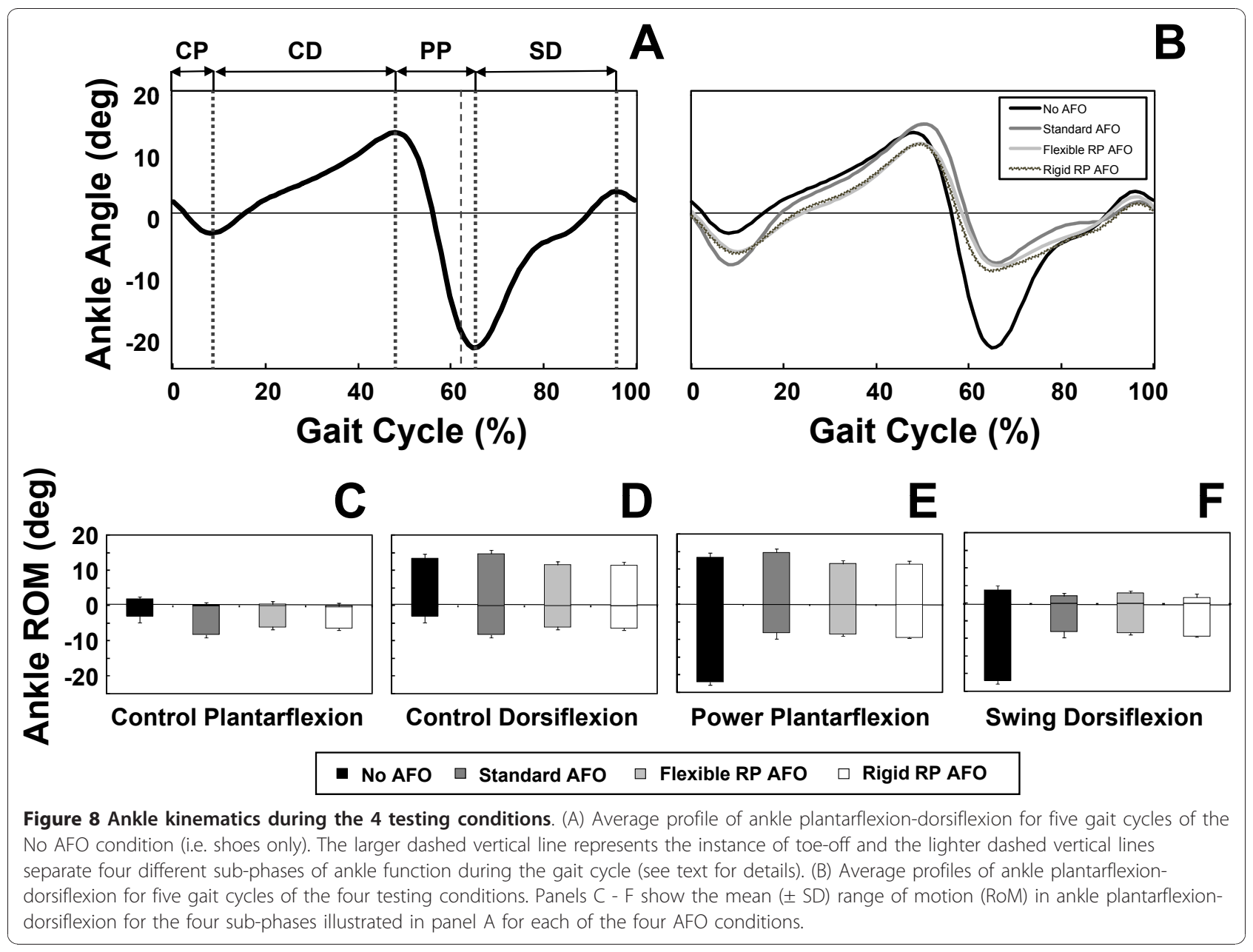



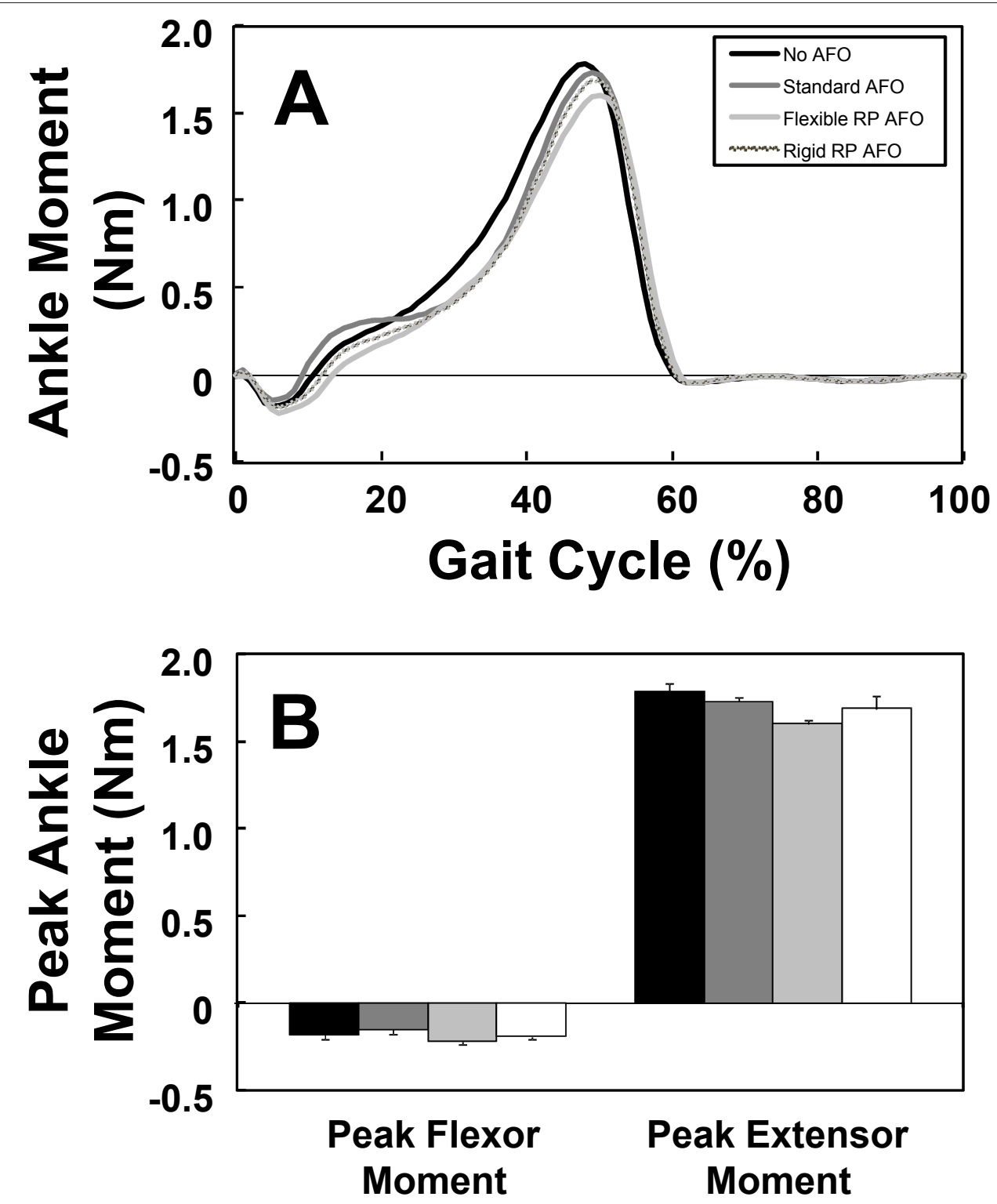

No AFO

Standard AFO

Flexible RP AFO

Rigid RP AFO

Figure 9 Ankle kinetics during the 4 testing conditions. (A) Average profiles of ankle flexor-extensor moments for five gait cycles of the four testing conditions. (B) Mean $( \pm \mathrm{SD})$ peak ankle extensor and flexor moments for the four testing conditions.

showed that this testing condition was associated with high ankle power; most likely because the rigid AFO provided resistance to bending that the subject had to overcome. Despite differences among AFO's, it was noted that the change in ankle power was still relatively small, and that increased material flexibility would have been likely to help improving performance.

\section{Conclusions}

In this paper, we presented a process to combine state of the art 3D scanning hardware and software technologies for human surface anatomy with advanced RP techniques so that novel custom made orthoses and rehabilitation devices can be rapidly produced. Two custom-fit AFOs were rapidly prototyped to demonstrate 

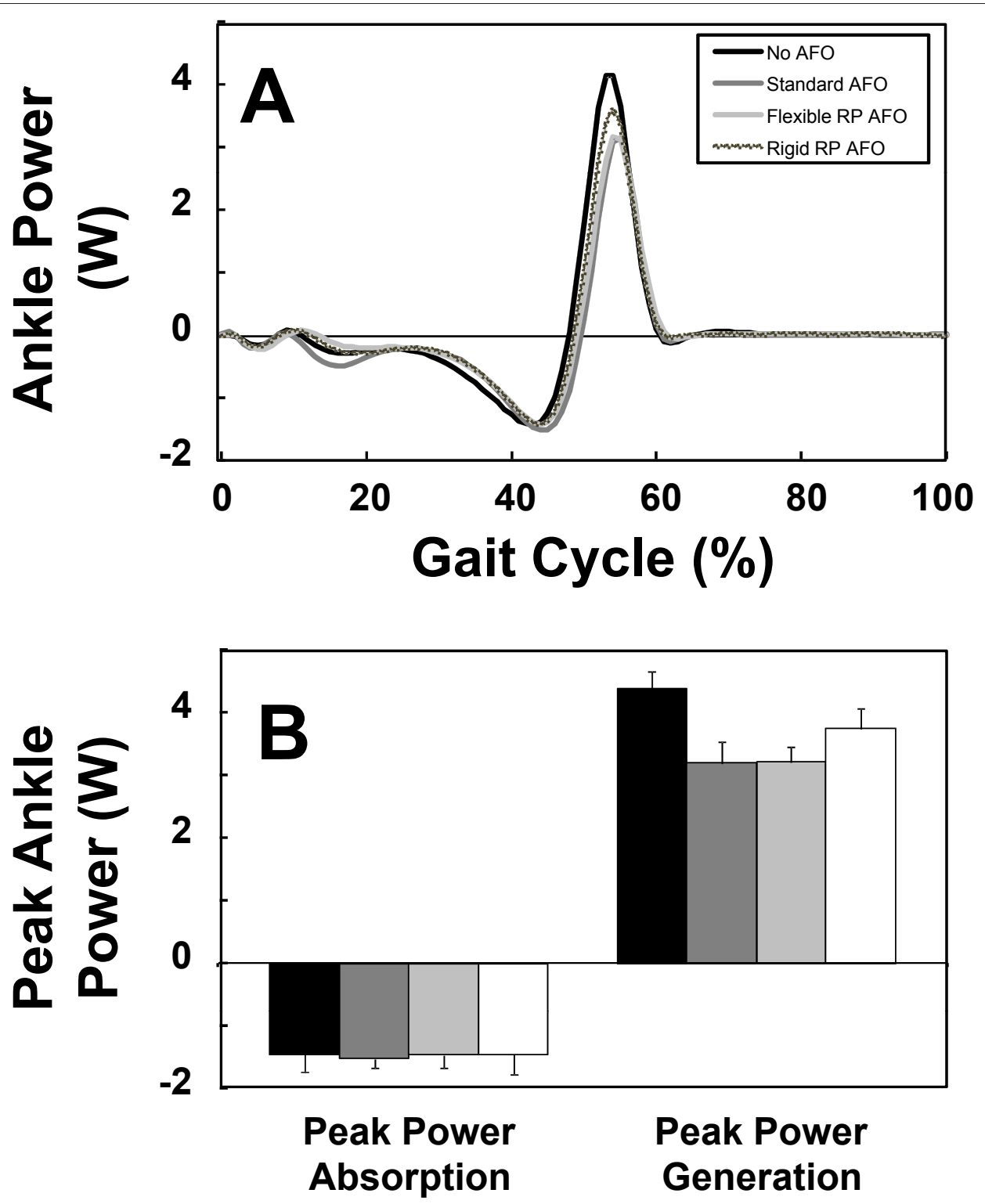

\section{No AFO \\ Standard AFO \\ Flexible RP AFO \\ Rigid RP AFO}

Figure 10 Ankle power during the 4 testing conditions. (A) Average profiles of ankle powers for five gait cycles of the four testing conditions. (B) Mean ( \pm SD) peak power absorption and power generation at the ankle for the four testing conditions.

the proposed process. Preliminary biomechanical data from gait analyses of one subject wearing the AFOs indicated that the RP AFOs can match the performance of the standard, prefabricated, polypropylene design. This new platform technology for developing custom-fit $\mathrm{RP}$ orthoses has the potential to provide increased freedom with geometric features, cost efficiencies and improved practice service capacities while maintaining very high quality-of-service standards. In the long run, this technology aims at bringing the manufacturing of orthoses from the current manual labor/expert craftsman's skills to a $21^{\text {st }}$ century computerized design process. The proposed technology has the potential for increasing the numbers of patients serviced per year per 
orthotist while reducing overall the orthosis fabrication cost and time.

\author{
Author details \\ ${ }^{1}$ Department of Mechanical \& Industrial Engineering, Northeastern University, \\ 360 Huntington Avenue, Boston, MA, 02115, USA. ${ }^{2}$ Department of Physical \\ Medicine and Rehabilitation, Harvard Medical School, Spaulding \\ Rehabilitation Hospital, 125 Nashua Street, Boston, MA, 02114, USA \\ ${ }^{3}$ Polymesh LLC, 163 Waterman Street Providence, RI 02906-3109. ${ }^{4}$ IAM \\ Orthotics \& Prosthetics, Inc., 400 West Cummings Park, Suite 4950, Woburn, \\ MA, 01801, USA. ${ }^{5}$ Harvard-MIT Division of Health Sciences and Technology, \\ 77 Massachusetts Ave., Cambridge, MA, 02139, USA.
}

\section{Authors' contributions}

$\mathrm{CM}, \mathrm{PB}, \mathrm{ML}$ : conceived the study and participated in the design and data analysis.

RGR, MLS, AC, KG, LG, SS: carried out the design, fabrication, testing of the RP AFOs.

BLP: participated in the testing of the RP AFOs and performed the data analysis.

JD, RD: participated in the design and data analysis.

All authors read and approved the final manuscript.

\section{Competing interests}

The authors declare that they have no competing interests.

Received: 1 April 2010 Accepted: 12 January 2011

Published: 12 January 2011

\section{References}

1. Chelule K, Coole T, Chesire D: Fabrication of medical models from scan data via rapid prototyping techniques. Proceedings of the 2000 Conference on Time Compression Technologies Cardiff International Arena, UK; 2000.

2. CC Kai, Meng CS, Ching LS, Teik LS, Aung SC: Facial prosthetic model fabrication using rapid prototyping tools. Integrated Manufacturing Systems 2000, 11(1):42-53.

3. Hieu LC, Zlatov N, Sloten JV, Bohez E, Khanh L, Binh PH, Oris P, Toshev Y: Medical rapid prototyping: applications and methods. Assembly Automation 2005, 25(4):284-292.

4. Zollikofer CPE, Ponce de Leon MS: Tools for rapid prototyping in the biosciences. IEEE Computer Graphics and Applications 1995, 15(6):48-55.

5. Noorani R: Rapid Prototyping: Principles and Applications John Wiley \& Sons Inc. Hoboken;; 2006.

6. Webb PA: A review of rapid prototyping (RP) techniques in the medical and biomedical sector. Journal of Medical Engineering \& Technology 2000, 24(4):149-153.

7. Sinn DP, Cillo JE, Miles BA: Stereolithography for craniofacial surgery. The Journal of Craniofacial Surgery 2006, 17(5):869-875.

8. Chua CK, Leong KF, KH Tan, Wiria FE, Cheah CM: Development of tissue scaffolds using selective laser sintering of polyvinyl alcohol/ hydroxyapatite biocomposite for craniofacial and joint defects. Journal of Material Science: Materials in Medicine 2004, 15:1113-1121.

9. Herbert N, Simpson D, Spence WD, W lon: A preliminary investigation into the development of 3-D printing of prosthetic sockets. Journal of Rehabilitation Research \& Development 2005, 42:141-146.

10. Rogers B, Bosker GW, Faustini MF, Walden G, Neptune RR, Crawford RH: Variably Compliant Transtibial Prosthetic Socket Fabricated Using Solid Freeform 'a case study'. Journal of Prosthetics and Orthotics 2008, 20(1):1-7.

11. Kumar V, Bajcsy R, Harwin W, Harker P: Rapid design and prototyping of customized rehabilitation aids. Communications of the ACM 1996, 39(2):55-61.

12. Milusheva $S$, Tochev D, Stefanova $Y, Y$ Toshev $Y$ : Virtual models and prototype of individual ankle foot orthosis. Proceedings of ISB XXth Congress - ASB 29th Annual Meeting Cleveland, Ohio; 2005, 227.

13. Milusheva S, Tosheva E, Tochev D, Toshev Y: Personalized ankle foot orthosis with exchangeable elastic elements. Journal of Biomechanics 2007, 40(S2):S592.
14. Faustini MC, Neptune RR, Crawford RH, Stanhope SJ: Manufacture of passive dynamic ankle-foot orthoses using selective laser-sintering. IEEE Transactions of Biomedical Engineering 2008, 55(2):784-790.

15. Alimed Inc.: Type C-90 Superior Posterior Leaf Spring 2009 [http://www. alimed.com].

16. Technest Holdings, Inc:: 3D Imaging Products [http://www.genextech.com/ pdfs/3DImaging/Genex_3DSolutions_web.pdf].

17. 3D Systems Inc.: Products - SLA Systems - Viper Si2 2009 [http:// www.3dsystems.com/products/sla/viper/datasheet.asp].

18. Rapidform Inc.: The Standard Software for 3D Scanning 2009 [http://www. rapidform.com/]

19. 3D Systems Inc.: Products - Accura 40 SLA Resin 2009 [http:// www.3dsystems.com/products/datafiles/accura/datasheets/DSAccura_25_SL_material.pdf].

\section{doi:10.1186/1743-0003-8-1}

Cite this article as: Mavroidis et al:: Patient specific ankle-foot orthoses using rapid prototyping. Journal of NeuroEngineering and Rehabilitation 2011 8:1.

\section{Submit your next manuscript to BioMed Central and take full advantage of:}

- Convenient online submission

- Thorough peer review

- No space constraints or color figure charges

- Immediate publication on acceptance

- Inclusion in PubMed, CAS, Scopus and Google Scholar

- Research which is freely available for redistribution

Submit your manuscript at www.biomedcentral.com/submit
C) Biomed Central 